\title{
An Improved Two-Stage Optimization Procedure for Optimal Power Flow Calculation
}

\author{
A.V. Domyshev*, D.N. Sidorov, D.A. Panasetsky \\ Melentiev Energy Systems Institute of Siberian Branch of Russian Academy of Sciences, Irkutsk, Russia
}

\begin{abstract}
The paper focuses on the problem of optimal power flow. Minimized production and transmission costs were considered to design an optimal power flow search method based on the novel two-stage optimization procedure that takes into account the voltage and power constraints. As a result, the efficient optimum search method for the non-convex optimal power flow problem is proposed and implemented. The efficient L-BFGS-B optimization algorithm is employed to allow for the constraints. The stochastic modification of the global optimization algorithm of the Lipschitz function was used in the first stage to improve the L-BFGS-B method. Two illustrative examples on the IEEE 118-bus test scheme and real regional grid demonstrate the efficiency of the proposed two-stage optimization procedure. The possibility of taking into account the renewable sources in the proposed optimal power flow algorithm is briefly discussed
\end{abstract}

Index Terms - optimal power flow, power system control, power system modeling, renewable energy sources.

\section{INTRODUCTION}

Optimization of power system operation involves the determination of an optimal steady state in terms of minimized production and transmission costs. This paper proposes a two-stage optimization procedure taking into account voltage and power constraints. This is a nonconvex problem as demonstrated below. A method of global and local optimum search is suggested to find a solution to the optimal power flow (OPF) problem. The

\footnotetext{
${ }^{*}$ Corresponding author.

E-mail: domyshev@isem.irk.ru
}

http://dx.doi.org/10.38028/esr.2020.01.0005

Received January 16, 2020. Revised March 20, 2020.

Accepted May 30, 2020. Available online July 31, 2020.

This is an open access article under a Creative Commons Attribution-NonCommercial 4.0 International License.

(C) 2020 ESI SB RAS and authors. All rights reserved. proposed method allows taking into account the constraints using the L-BFGS-B (Limit memory version of Broyden Fletcher Goldfarb and Shanno optimization method with Border constraints) optimization algorithm.

The power systems operation optimization under various constraints has been fairly well addressed by many authors in the literature. On the one hand, many methods were designed and applied including the gradient descent, Newton method, the interior points method, conjugate gradients, and Lagrange relaxation [1-4]. Various artificial intelligence (AI) methods have been also widely used during the last decades. These are multi-agents systems, artificial neural networks, genetic algorithms, evolutionary programming, fuzzy logic-based control, particles swarm optimization, and many others [5-7]. On the other hand, many flexible control strategies have been recently proposed to cope with flexible modern power systems with renewable generation and storage [8-9].

It is worth noting that the efficient optimization of power flow remains a challenging problem especially for the power systems with a high share of renewables. Moreover, unstable low-frequency inter-area oscillations [10], caused by high level of renewable energy penetration, may have a severe negative effect on the objective function of optimal power flow and maximum power transfer.

The following challenges are under attacks most often:

1. Minimization of energy losses with the following control actions:
a. Active and reactive power redistribution;
b. The tap-changers adjustment;
c. The shunt reactors and capacitor banks states' adjustment;

2. Change in overflows on transmission lines due to the FACTS devices.

3. Power flow adjustment for the operating conditions to meet voltage and flow constraints.

4. Optimization of active power generation according to economic criteria.

In this paper, our results [11] are further developed based on a new two-stage optimization procedure and the L-BFGS-B algorithm, namely: 
1. The global optimization and the local L-BFGS-B optimization methods are employed in the new software implementation of the proposed method. The flowchart of the generalized algorithm is presented in section $\mathrm{V}$.

2. More representative calculations on the same test schemes are carried out.

The paper is structured as follows. The problem statement is given in Section II. The non-convex OPF problem is addressed in Section III. Section IV proposes a stochastic global optimization algorithm to improve the conventional L-BFGS-B method. Section V describes the application of the L-BFGS-B method. Case studies on two test schemes are given in Section VI. Section VII describes further research, in particular, a study on the possibility of including renewable sources in the optimization. Finally, Section VIII presents the concluding remarks.

\section{PROBLEM STATEMENT}

The optimal power flow methods most often use only one criterion either minimization of generation cost or minimization of losses. This paper employs a complex optimization criterion and takes into account the cost of generation, losses in a grid, and voltage magnitude deviation [36]. Generation cost here is understood not as actual cost expressed in cash equivalents, but as a dimensionless quantity taking into account the loading preferences of various generators. As a result, the OPF problem is formulated as follows:

$$
\min f\left(p_{g}, u_{g}, k_{t}\right)
$$

where the objective function $f$ is

$$
\begin{aligned}
& f=\sum_{i \in G}\left(c_{i 2} p_{g i}^{2}+c_{i 1} p_{g i}+c_{i 0}\right)+ \\
& +c_{\Delta P} \sum_{j \in B} \Delta p_{j}+c_{U} \sum_{k \in N} \Delta u_{k}{ }^{2}
\end{aligned}
$$

The control parameters in the OPF problem (2) are as follows: $p_{g}, u_{g}, k$ are the generation of active power, the voltage at PU buses and transformer ratios, respectively. Here $G$ is a set of generator buses, $B$ is a set of branches, $N$ is a set of buses, $p_{g i}$ is the active power of the $i$-th generator, $\Delta p_{j}$ is power losses on branch $j, \Delta u_{k}$ is a voltage magnitude deviation at bus $k$.

The objective function is adjusted as follows: $\mathrm{c}_{\mathrm{i} 2}, \mathrm{c}_{\mathrm{i} 1}$ , $\mathrm{c}_{\mathrm{i} 0}$ are constants adjusting the generation cost; $\mathrm{c}_{\Delta \mathrm{P}}$ is a constant which takes into account the transmission losses; $c_{u}$ is a constant for voltage deviations on buses.

The constraints on control parameters are as follows:

$$
\begin{array}{ll}
p_{g i}^{\min } \leq p_{g i} \leq p_{g i}^{\max }, & \forall i \in G \\
u_{g i}^{\min } \leq u_{g i} \leq u_{g i}^{\max }, & \forall i \in G \\
k_{t i}^{\min } \leq k_{t i} \leq k_{t i}^{\max }, & \forall i \in T
\end{array}
$$

Here $G$ is a set of generator buses involved in optimization, $T$ is a set of transformer branches involved in optimization. Assume that the dependent parameters $\Delta p_{j}$ are power losses in the transmission lines and $\Delta u_{k}$ are voltage deviations on buses. These parameters are calculated using the electric network system of equations. An original modification of the Newton-Raphson method with the selection of an optimal step was involved to calculate the power flow. The power flow calculation process takes into account other constraints, such as the $\mathrm{P} / \mathrm{Q}$ curves of the generators. In this method, the bus voltage equations are written in Cartesian coordinates. This method was proposed in [12] and developed in [13]. Load flow equations in Cartesian coordinates are:

$$
s=f_{s}(u) \text {, }
$$

Where $u=\left[u_{r 1}, u_{i 1}, \ldots, u_{r n}, u_{i n}\right]$ is the vector of real and imaginary parts of bus voltages; $s=\left[P_{1}, Q_{1}, P_{2},\left|V_{2}\right|^{2}, \ldots,\left|V_{s}\right|^{2}, \delta_{s}\right]$ is the vector of independent parameters: active and reactive power of PQbuses; active power and voltage at PU-buses; voltage and phases at slack buses. Electric network equations can be written in a matrix form as follows:

$$
s=-\operatorname{diag}(u) Y u^{T} .
$$

The matrix of coefficients $Y$ is formed based on the conductivities between buses and shunt conductivities at buses [13].

\section{NONCONVEX OPTIMIZATION}

The necessary Karush-Kuhn-Tucker conditions are in the core of many optimization methods for power systems operation optimization. Such necessary conditions can guarantee only a locally optimal solution in real-world applications such as the non-convex optimum power flow problem $[14,15]$. It can be argued that the non-convexity of the optimization problem is determined by the nonlinear dependence on physical parameters (including power and voltage), and by the power flow parameters constraints.

The state-of-the-art studies have paid much attention to the development of high performance algorithms. Let us briefly outline the advances in this field. The nonlinear interior point algorithms are proposed in $[14,15]$. The origin of the non-convexity of the given optimization problem is shown in $[18,19]$ where the limits of power flow stability are taken into account. In this case, the stability at each iteration of optimization is an additional constraint. The convex relaxation is proposed for solving the optimal power flow problem in $[14,20]$. However, this approach may not be suitable for the large dimension schemes. As shown in [14], the EPS operation optimization is an NPhard problem with the objective function usually assumed to be convex $[14,21]$ for the sake of simplicity.

Thus, many authors assume that the non-convexity of the optimal power flow problem arises only because of the need to take into account different constraints, for example, constraints imposed by the system operator to control the current power flow in EPS. This assumption was studied in the framework of this paper. A search for a local optimum for the complex networks was made, starting with various randomly obtained initial states within the feasible region of the power flow parameters. 

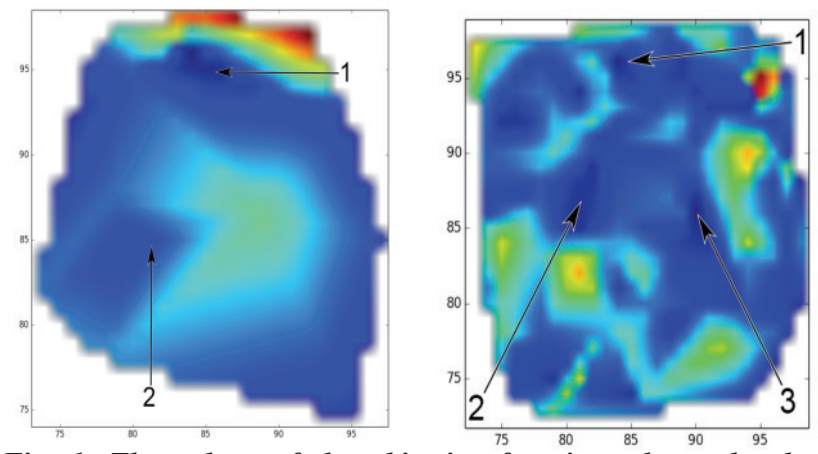

Fig. 1. The values of the objective function, shown by the intensity of the color (numerals denote the areas of local optima): a) for random search without optimization; b) with subsequent optimization.

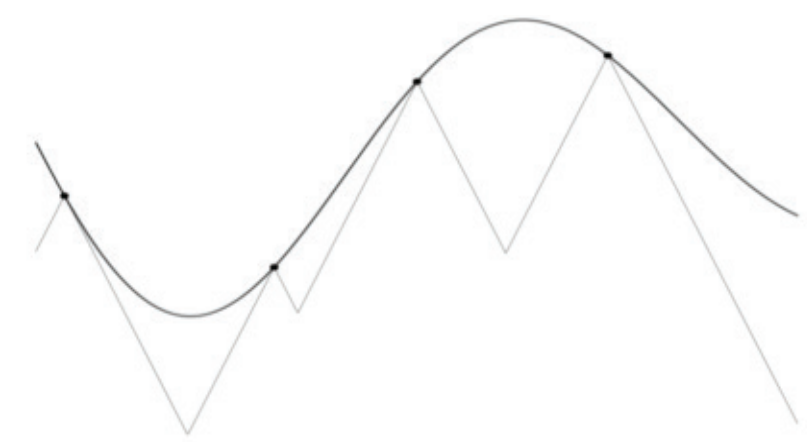

$x$

Fig. 2. AdaLIPO Algorithm illustration.

The calculation was carried out on the model of the Irkutsk region power system shown in Figure 4. The problem was simplified. In the objective function, we took into account only the terms responsible for network losses and voltage deviations. As control actions, only transformation ratios were used. At each random search iteration, random values of the control parameters from the feasible region were selected. After that, the power flow was calculated. In the case of convergence, the results were used as a starting point for local optimization by gradient descent algorithm. As can be seen, even in such a simplified case, the objective function is not convex.

The values of the objective function in the n-dimensional space of the control parameters were obtained, where $n$ is the number of the control parameters. For clarity, Figure 1 shows the values of the objective function in the crosssection of the two most variable control parameters.

The calculations revealed the presence of several local optima. Therefore, to determine the scope of the local search it is necessary to employ the methods of global optimization. This will be addressed further.

\section{GLOBAL OPTIMIZATION ALGORITHM}

To find the global optimum, the search for regions of the local optimum is performed using the stochastic global optimization algorithm of the Lipschitz function (LIPOP), based on LIPO algorithm proposed in [22].

Since the discrete changes in EPS control parameters are assumed to be continuous, the optimization problem (1) can be considered as the optimization problem for the Lipschitz function.

In the AdaLIPO algorithm[22], a random sampling of points $X_{i}$ from the feasible region of control parameters is made and the lower bound of the function is estimated from the closest points (as shown in Fig.2) using the Lipschitzness coefficient $l$ :

$$
x \rightarrow \max _{i=1 . . t} f\left(X_{i}\right)-l \cdot\left\|x-X_{i}\right\|_{2},
$$

Where $X_{i}$ is the control parameters vector.

The coefficient is refined by the values of the objective function, as the maximum among the test points:

$$
l=\max _{i \in T, j \in T, i \neq j} \frac{\left|f\left(X_{i}\right)-f\left(X_{j}\right)\right|}{\left\|X_{j}-X_{i}\right\|_{2}}
$$

In order to increase the density of the test points in the region with a large gradient of the objective function and to reduce the number of tests in the region with small gradient values, a random search algorithm with the probability depending on the gradient is applied. The power flow is calculated only if the random sample $p \in[0,1]$ is less than the relative magnitude of the gradient $g_{i}$ :

$$
p_{i}<\frac{\left\|g_{i}\right\|}{\min _{i \in T}\left\|g_{i}\right\|}
$$

Here we call the modification of the LIPO algorithm LIPOP (P stands for "probability"). The Jacobian of the objective function was calculated in (7) by the numerical algorithm using Richardson extrapolation [23, 24].

The solution obtained by the global optimization method LIPOP can act as an initial approximation for performing local optimization by more accurate methods. Thus, the global optimization of the power flow can be carried out in two stages. In the first stage, the search is performed for the region with the least value of the objective function by global search in the feasible region. The second stage solves the problem of finding a local optimum with more precise methods.

\section{APPLICATION OF THE BFGS METHODS}

In this paper, the method based on the L-BFGS-B algorithm [25-27], which is a development of the BFGS method, is proposed as a method for finding a local optimum. This method is a quasi-Newton method that takes into account the constraints on the control parameters.

In this case, the constraints on power flow parameters are allowed for in two different ways. Control parameter constraints are taken into account in a standard way in the form of boundary conditions: $l \leq x \leq u$. Constraints on dependent power flow parameters are taken into account in the form of barrier functions, the values of which will be included in the general optimization objective function and will be calculated in the process of power flow calculation 
at each iteration of the optimization for the fixed values of the control parameters. The logarithmic functions are proposed to be used as barrier functions by analogy with the interior point method:

$$
F(x)=-\sum\left(-b_{i}(x)\right)
$$

where $b_{i}(x) \leq 0, i=1, \ldots, m$ are dependent parameters constraints; $m$ is the number of constraints.

This approach allows us to divide the optimization problem into the power flow calculation and the optimization problem itself. Due to the decomposition of the optimization problem into the power flow calculation and optimization, on the one hand, the constraints are simplified as follows $l \leq x \leq u$, on the other hand, in the framework of the power flow calculation at each iteration we can use complex network models including DC-lines, active filters, reactive power compensators, FACTS, and other devices.

As a result, the algorithm for finding the local optimum by the L-BFGS-B method is as follows:

1. At each step of the iterative process, we have the control vector $x_{k}$, the objective function value $f\left(x_{k}\right)$ and the gradient of the objective function $g_{k}$ calculated at point $x_{k}$.

2. The gradient projection method is used to meet the constraints $l \leq x \leq u$.

3. The quadratic model of function $f$ is calculated as follows:

$$
\begin{aligned}
& m_{k}(x)=f\left(x_{k}\right)+g_{k}^{T} \cdot\left(x-x_{k}\right)+ \\
& +\frac{1}{2}\left(x-x_{k}\right)^{T} \cdot B_{k} \cdot\left(x-x_{k}\right),
\end{aligned}
$$

where $B_{k}$ is the Hessian approximation obtained by the quasi-Newton algorithm L-BFGS [28, 29]. Next, we determine a piecewise linear function that coincides in the direction with the antigradient and takes into account the constraints:

$$
x(t)=P\left(x_{k}\right)-t \cdot g_{k}, l, u
$$

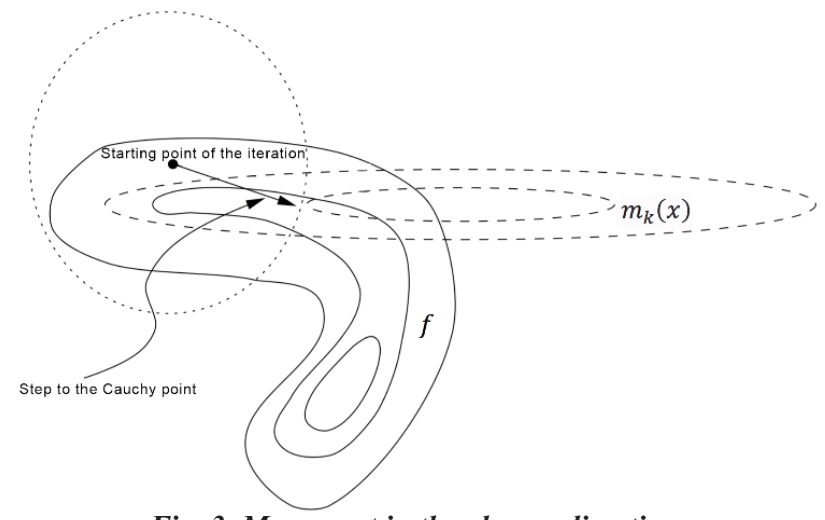

Fig. 3. Movement in the chosen direction.

Where

$$
P(x, l, u)_{i}=\left\{\begin{array}{c}
l_{i}, \quad \text { if } x_{i}<l_{i} \\
x_{i}, \quad \text { if } x_{i} \in\left[l_{i}, u_{i}\right] \\
u_{i}, \text { if } x_{i}>u_{i}
\end{array}\right.
$$

The next step is the calculation of Cauchy point as the local minimum of the model function for the selected piecewise- linear direction (as shown in Figure 3):

$$
x_{c}=\underset{x}{\operatorname{argmin}} m_{k}(x(t)),
$$

The iterative process continues while the change in the control vector in the direction of optimization is greater than the selected threshold $\left\|\mathrm{x}_{\mathrm{k}}-\mathrm{x}_{\mathrm{k}-1}\right\|>\varepsilon$.

In the process of optimization, there can be situations when the power flow does not exist, for example, due to the power flow constraints. In this case, it is necessary to find the limiting state along the given trajectory and set new constraints.

The movement along the trajectory is performed by dividing the increments of the vector of the control actions in half. The quadratic model (9) in this case is written as:

$$
m_{k}(x)=f\left(x_{k}\right)+g_{k}^{T} \cdot \Delta x+\frac{1}{2} \Delta x^{T} \cdot B_{k} \cdot \Delta x
$$

here $\Delta x=\frac{x-x_{k}}{2 \alpha}, \alpha$, is the multiplicity of the step division.

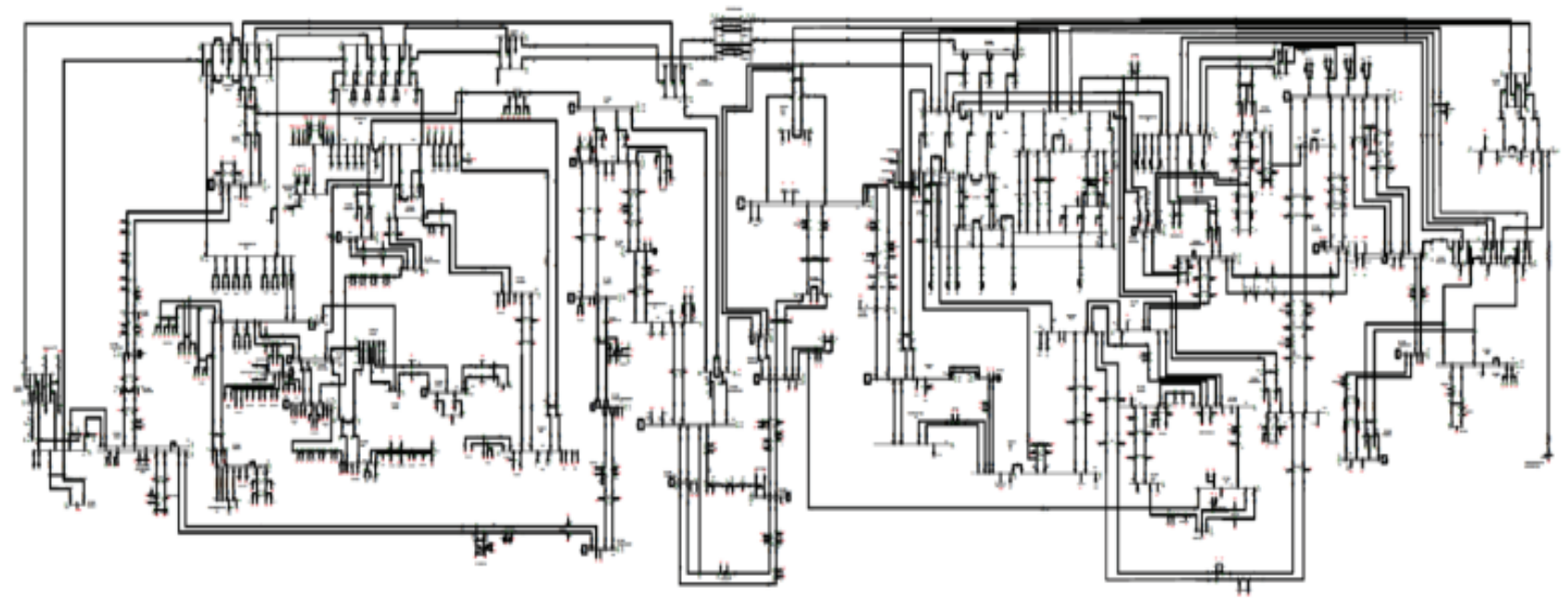

Fig. 4. Real power grid. 


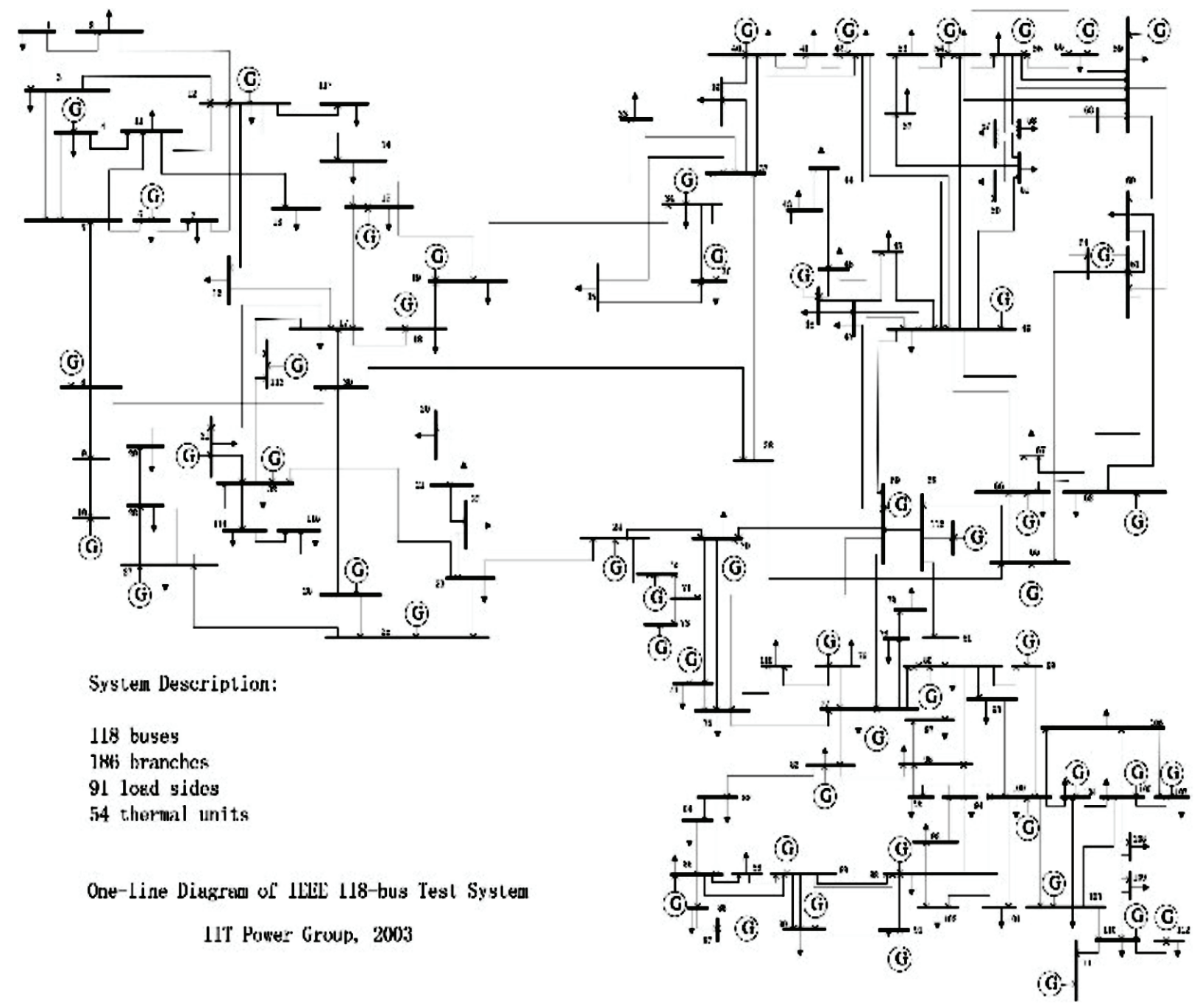

Fig. 5. IEEE 118 bus test system.

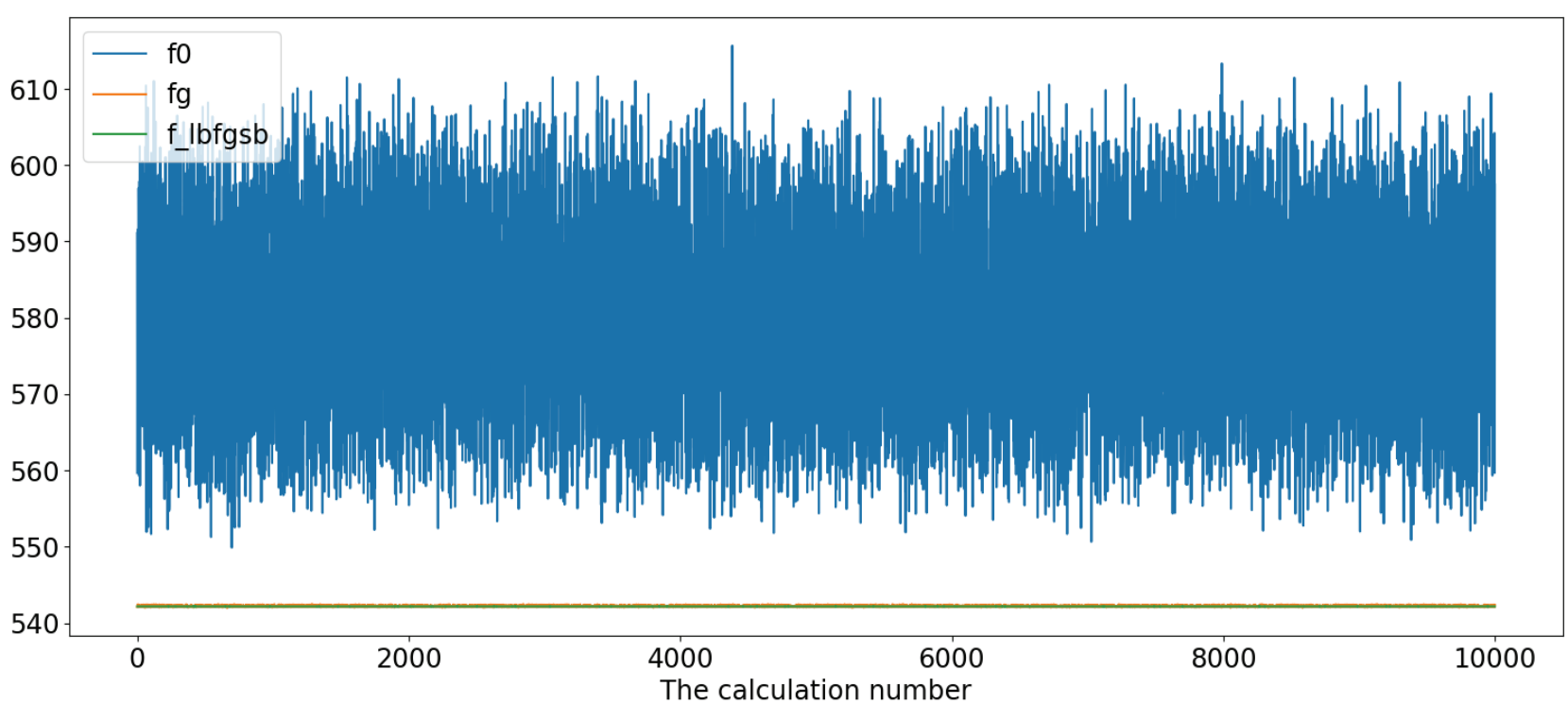

Fig. 6. Calculation results on the IEEE 118-bus test system. $f_{0}$ is an initial value of the objective function in p.u.; $f_{g}$ is the objective function after gradient descent in p.u.; $f_{\text {lbfgsb }}$ is the objective function after L-BFGS-B optimization in p.u. The ordinate axis is a serial number in a series of calculations with different initial states. 


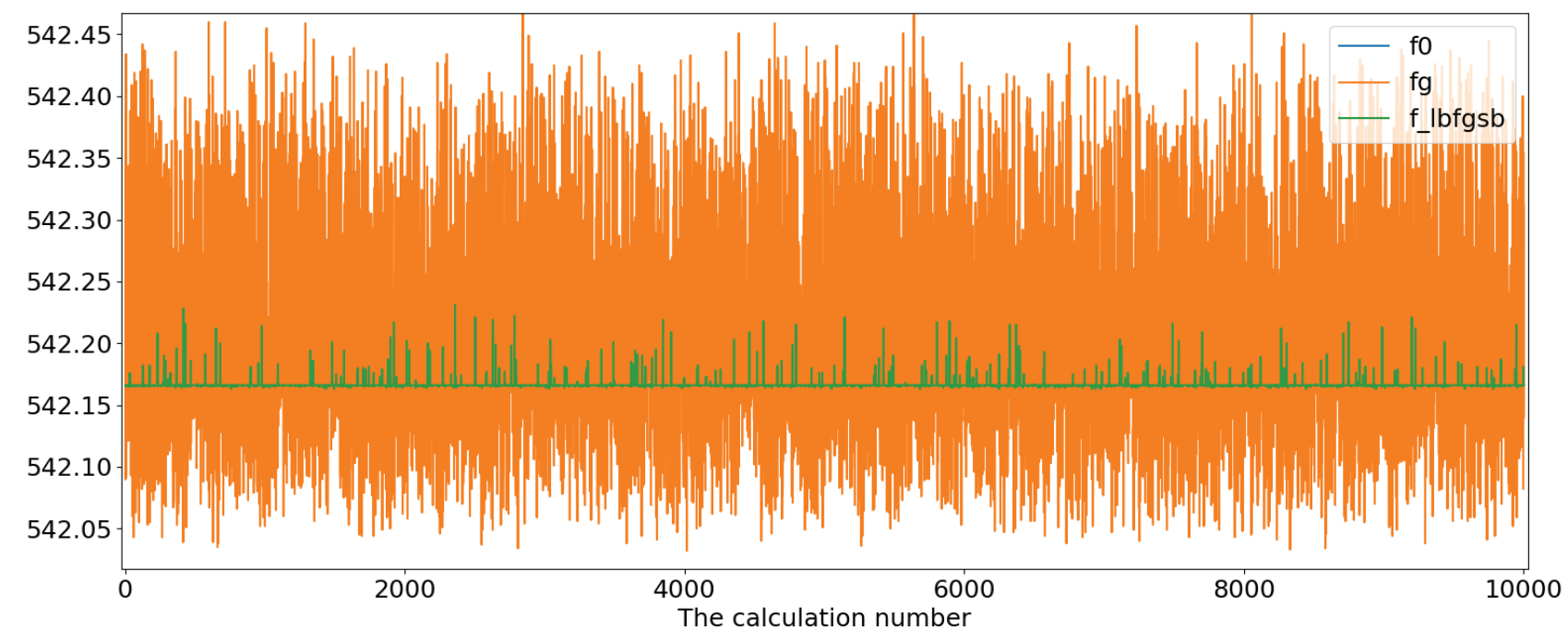

Fig. 7. Objective function after optimization on the IEEE 118-bus test system. $f_{g}$ is the objective function after gradient descent in p.u.; $f_{\text {lbfgsb }}$ is the objective function after L-BFGS-B optimization in p.u. The ordinate axis is a serial number in a series of calculations with different initial states.

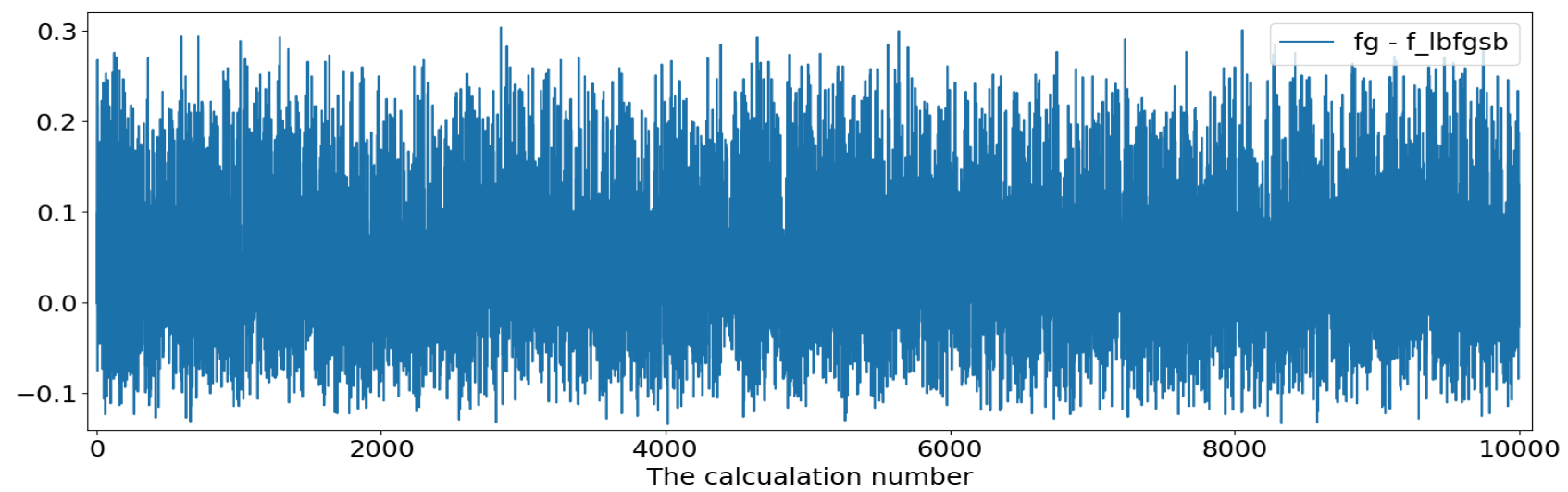

Fig. 8. Difference between objective functions in gradient descent and L-BFGS-B methods on the IEEE 118-bus test scheme. The ordinate axis is a serial number in a series of calculations with different initial states.

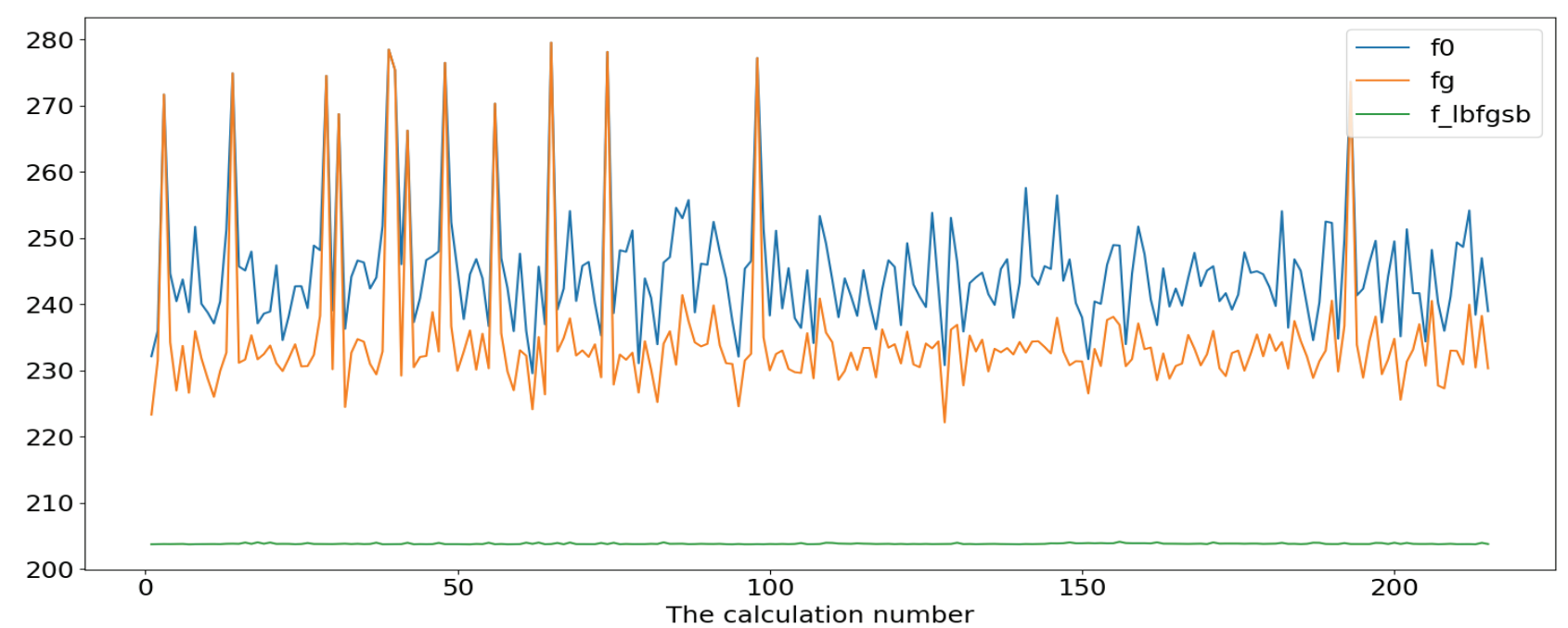

Fig. 9. Calculation results on Irkutsk region network model without constraints. $f_{0}$ is the initial value of the objective function in p.u.; $f_{g}$ is the objective function after gradient descent in p.u.; $f_{\text {lbfgs }}$ is the objective function after L-BFGS-B optimization in p.u. The ordinate axis is a serial number in a series of calculations with different initial states. 


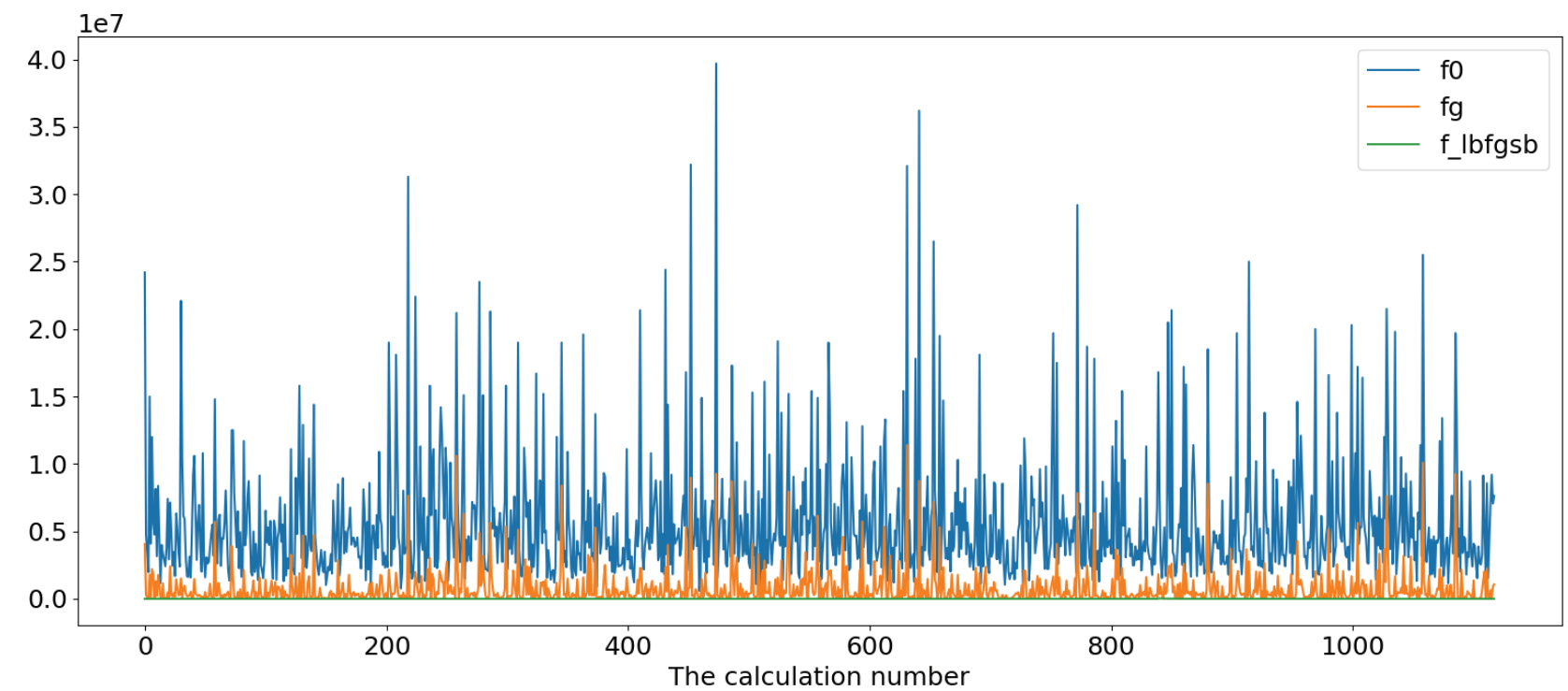

Fig. 10. Calculation results on the Irkutsk region network model with constraints. $f_{0}$ is the initial value of the objective function in p.u.; $f_{g}$ is the objective function after the gradient descent in p.u.; $f_{\text {lbfgsb }}$ is the objective function after L-BFGS-B optimization. The ordinate axis is a serial number in a series of calculations with different initial states.

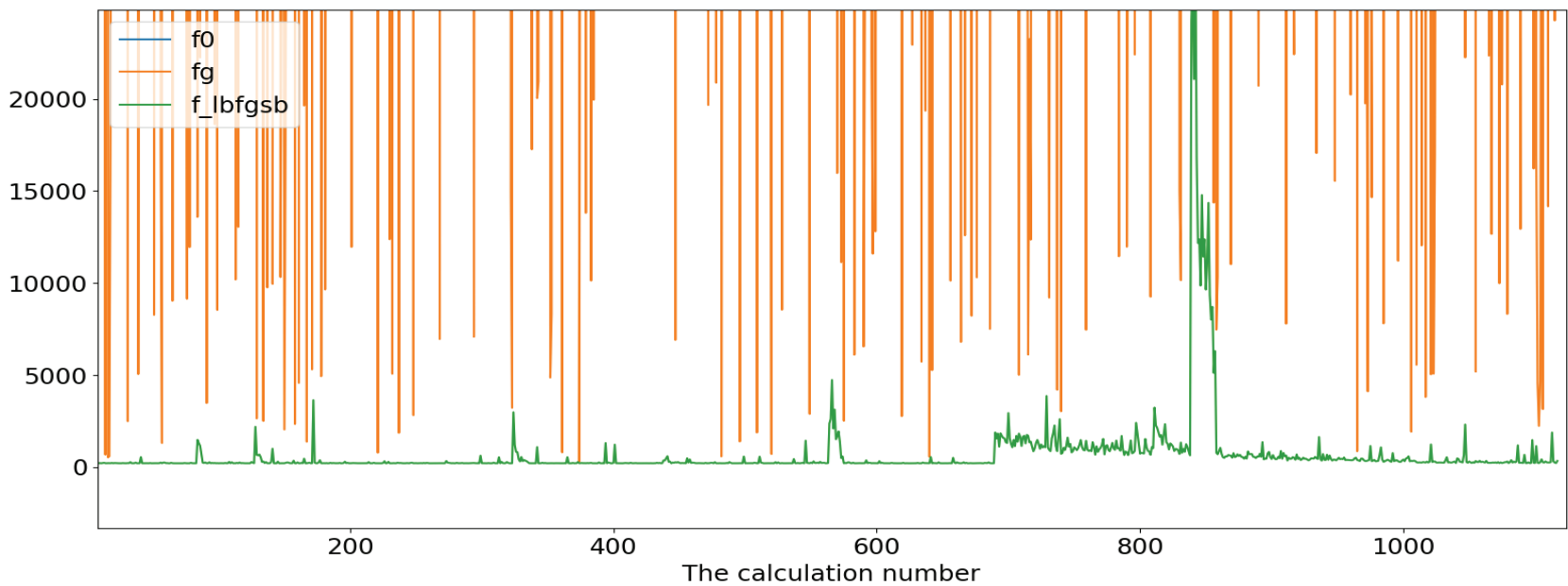

Fig. 11. A scaled plot of calculation results on the Irkutsk region network model with constraints. $f_{g}$ is the objective function after the gradient descent in p.u.; $f_{\text {lbfgsb }}$ is the objective function after L-BFGS-B optimization. The ordinate axis is a serial number in a series of calculations with different initial states.

The resulting flowchart of the proposed algorithm is as follows:

1. Perform global LIPOP optimization restricted by the number of iterations:

1.1. Sample randomly control parameters from the feasible region according to (7).

1.2 Calculate the objective function (1).

1.3 Calculate the objective function.

1.4 Save points with the lowest value of the objective function in the restricted vector of global optimization results.

2. Perform local optimization with L-BFGS-B for every point from the vector of global optimization results.

3. Select the optimal point from the results of local optimization.

\section{CASE STUDY}

The proposed optimization scheme was tested on the IEEE 118- bus test system (shown in Figure5), and on the model of the Irkutsk region network (shown in Figure 4).

About 10000 series of calculations were performed. A random value of the vector of the control parameters is selected as a starting point within the feasible range where it was possible to calculate the steady state. The ordinate axis in the following Figures represents the values of the objective function in per units. The abscissa axis corresponds to a series of calculations from different starting points.

The results with a well-chosen step in the gradient descent optimization method on the IEEE 188-bus test scheme are quite close to the results of L-BFGS-B 
algorithm (Figure 6), however, the calculation results obtained using L-BFGS-B are more stable (Figure 7) and on average give a $4 \%$ better result (Figure 8 ).

A more vivid result is observed on the model of real electrical networks. If the optimization is performed on a model without constraints, the result of the L-BFGS-B algorithm is stable and several times better than the gradient descent (Figure 9). If the algorithm takes into account the constraints on the bus voltage, the application of the L-BFGS-B algorithm becomes even more effective (Figures 10-11). On a larger scale plot (Figure 11), it can be seen that L-BFGS-B method gives adequate results with a minimum objective function for almost all initial conditions, while the gradient descent for most of the initial states only slightly improves the objective function relative to the initial one (Figure 11).

This difference in the results can be explained by the fact that the model of the real power system has worse convergence of the initial state, and the gradient descent here is more sensitive to the initial descent step.

The speeds of the optimization algorithms are comparable. Thus, in the scheme of a real electrical network, the average operating time of the gradient optimization method is about $120 \mathrm{msec}$, and that of the L-BFGS-B algorithm is about $500 \mathrm{msec}$.

\section{FURTHER STUDIES}

The development of micro-grids and renewables requires total rethinking of the EPS steady-state optimization. Wind and solar power plants are the major renewable energy sources (RES). The development of reliable power systems with high penetration of renewables is one of the challenges in modern power engineering. Such systems heavily depend on the weather conditions, which can be taken into account in the OPF problem in two different ways. The first approach is the generation forecasting based on the meteorological forecasts. In this approach, the optimization problem is divided into the classic problem of the optimal state search based on the given constraints and the problem of states prediction [7, 30, 31]. The second approach is based on the probabilistic calculation of the optimal power flow. In this case, the RES generation is given by the models that take into account the time-dependent probabilistic characteristics of generation as well as load. This is not only due to the variability of the traditional residential and industrial loads but also because the load can be active and can be influenced by the local RES such as small wind turbines and PVs (so-called electricity prosumers load) and storages [8].

The load model is usually given by a normal distribution with an average value and a standard deviation. The model of the wind power plant uses the Weibull distribution [31] to determine the probability of the wind speed, which is used to calculate the generation distribution of the wind power plant. The power distribution of the solar power plant is calculated using the beta-distribution function describing the solar irradiance $[5,33]$.

The probabilistic models of RES can be used to obtain the time distribution of generation as the function of time and installed generation capacity $\mathbb{P}_{g}\left(t, P_{g}\right)$. In this case, the objective function $f(\cdot)$ in (1) is as follows:

$$
\begin{aligned}
& \sum_{i \in G}\left(c_{i 2} p_{g i}^{2}+c_{i 1} p_{g i}+c_{i 0}\right)+c_{\Delta P} \sum_{j \in B} \Delta p_{j} \\
& +c_{U} \sum_{k \in N} \Delta u_{k}^{2} \\
& +\sum_{r \in R} c_{g r} \mathbb{P}_{g r}\left(t, P_{g r}\right) P_{g r} .
\end{aligned}
$$

The vector of independent parameters in the network equations (4) includes the power of RES generation and loads, given the corresponding probability distributions:

$$
\begin{gathered}
s=\left[\mathbb{P}_{l}\left(t, P_{l}\right) p_{l}, q_{l}, \mathbb{P}_{g}\left(t, P_{g}\right) p_{g},\right. \\
\left.q_{g}, p_{g}, u_{g}, \delta_{g}\right]^{T} .
\end{gathered}
$$

The optimal power flow calculations take into account the probabilities. Such calculations are performed using the two-point estimation method proposed by Verbic [34]. The essence of this method is as follows. For each value of generation and load given by the probability, two optimal power flows corresponding to values below and above the average are computed. The remaining probabilistic variables are selected as the mean value. Based on these two points, the vector of control parameters is estimated for each probability value.

The calculation of the optimal power flow, considering the probabilities, requires a significant number of deterministic optimal flow distribution calculations. Finding an optimal power flow for a certain time range is a more complicated problem. In this case, the dynamic optimization problem can be formulated as follows:

$$
\begin{aligned}
& \min f\left(t_{0}, T, x\right)=\int_{t_{0}}^{t_{0}+T} f_{p}(t, x) d t \\
& +f_{t}\left(\left\|x_{t_{0}-T}-x_{t_{0}+T}\right\|\right),
\end{aligned}
$$

Where $t_{0}$ is the initial time, $\mathrm{T}$ is the optimization time horizon, $f_{p}$ is the objective function calculated for each moment by the method of probabilistic optimal flow distribution, $f_{i}$ is the cost function of the transition from the initial state to the optimal one, determined as:

$$
f_{t}(x)=c_{t} \sum_{x \in X} 1, x>0 .
$$

Thus, the method of deterministic optimization is used in each stage of dynamic and probabilistic optimization.

\section{CONCLUSION}

The paper proposes an efficient two-stage optimization procedure based on the stochastic modification of the global optimization algorithm of the Lipschitz function and the L-BFGS-B method. The L-BFGS-B algorithm has a super-linear convergence rate. The complexity of the 
designed optimization algorithm is $O\left(n_{2}\right)$.

The decomposition of the calculation of the optimal power flow for the steady-state calculation problem and the optimization algorithm itself made it possible to efficiently perform optimization without complicating the optimal power flow calculation.

The presented optimization technique was implemented in the ANARES software [35]. The calculations on the models of the Irkutsk regional network revealed the following advantages. In comparison with the gradient method, the L-BFGS-B method of local optimization does not require to set an optimization step. The global optimization makes it possible to reach the global minimum of the objective function in a feasible region of the control parameters.

\section{ACKNOWLEDGMENTS}

This research has been carried out within the program of fundamental research of Siberian Branch of the Russian Academy of Sciences and within the framework of the research projects III.17.3.1, III.17.4.2, No. AAAA-A17-117030310442-8, No. AAAA-A17-117030310438-1. The authors are thankful to anonymous reviewers for their useful comments to improve the presentation and quality of the paper.

\section{REFERENCES}

[1] Yu. Nesterov, A. Nemirovski, "Interior-point aplynomial algorithms in convex programming," SIAM Studies in Applied Mathematics, vol. 13. 1993.

[2] Yu. Nesterov, "Introductory lectures on convex optimization: A basic course," Springer, 2013.

[3] S. Wright, J. Nocedal, "Numerical optimization," Springer Science, 2006.

[4] V.I. Zorkaltsev, "Dual interior points algorithms," Russian Mathematics, vol. 55, no. 4, pp. 26-43, 2011.

[5] J.A. Momoh, "Electric power system applications of optimization," CRC Press, 2008.

[6] J.A. Momoh, R. Adapa, M.E. El-Hawary, “A review of selected optimal power flow literature to 1993. I. Nonlinear and quadratic programming approaches," IEEE transactions on power systems, vol. 14, no. 1, pp. 96-104, 1999.

[7] F. Liu, L. R. Li, Y. Li, Y. Cao, D. Panasetsky, D. Sidorov, "Short-term wind power forecasting based on TS fuzzy model", In 2016 IEEE PES AsiaPacific Power and Energy Engineering Conference (APPEEC), pp. 414-418. IEEE, 2016.

[8] Y.Li, L. He, F. Liu, C. Li, Y. Cao, M. Shahidehpour, "Flexible voltage control strategy considering distributed energy storages for the dc distribution network," IEEE Transactions on Smart Grid, vol. 10, Issue: 1, Pages: 163 - 172, Jan. 2019.

[9] Y. Li, L. He, F. Liu, Y. Tan, Y. Cao, L. Luo, M. Shahidehpour, "A dynamic coordinated control strategy of WTG-ES combined system for short-term frequency support," Renewable Energy, vol. 119, pp 1-11, 2018.
[10] D. Sidorov, D. Panasetsky, and V. Šmádl, "Nonstationary autoregressive model for on-line detection of inter-area oscillations in power systems," 2010 IEEE PES Innovative Smart Grid Technologies Conference Europe (ISGT Europe), Gothenberg, 2010, pp. 1-5.

[11] A. Domyshev, D. Sidorov, D. Panasetsky, Y. Sun, P. Ju, and F. Wu, "Optimal Power Flow Calculation Using BFGS-Based Optimisation Scheme," Proc. Of 2nd IEEE Conference on Energy Internet and Energy System Integration (EI2), Beijing, pp. 1-6, 2018.

[12] Iwamoto, S., Y. Tamura, "A load flow calculation method for ill-conditioned power systems," IEEE transactions on power apparatus and systems, vol. 4, pp. 1736-1743, 1981.

[13] V.Z.Manusov, O.N.Shepilov, "Express methods of analysis of modes for assessing the reliability of electrical systems based on the Cartesian coordinate system," SEI, Irkutsk, pp. 168-175, 1989 (in Russian).

[14] J. Lavaei, S.H. Low, "Zero duality gap in optimal power flow problem," IEEE Transactions on Power Systems, vol.27, no.1, pp. 92-107, 2012.

[15] H. Wei et al. "An interior-point nonlinear programming for optimal power flow problems with a novel data structure," IEEE Transactions on Power Systems, vol. 13 , no. 3, pp. 870-877, 1998.

[16] W.M. Lin, C.H. Huang, T.S. Zhan, "A hybrid current-power optimal power flow technique," IEEE Transactions on Power Systems, vol. 23, no. 1, pp. 177-185, 2008.

[17] Q.Y. Jiang et al. "Power-current hybrid rectangular formulation for interior-point optimal power flow," IET generation, transmission \& distribution, vol. 3, no. 8, pp. 748-756, 2009.

[18] D. Gan, R.J. Thomas, R.D. Zimmerman, "Stabilityconstrained optimal power flow," IEEE Transactions on Power Systems, vol.15, no. 2, pp. 535-540, 2000.

[19] H.R. Cai, C.Y. Chung, K.P. Wong, "Application of differential evolution algorithm for transient stability constrained optimal power flow," IEEE Transactions on Power Systems, vol.23, no.2, pp.719-728, 2008.

[20] X. Bai et al. "Semidefinite programming for optimal power flow problems," International Journal of Electrical Power \& Energy Systems, vol. 30, no.6-7, pp 383-392, 2008.

[21] S.H. Low, "Convex relaxation of optimal power flow-Part I: Formulations and equivalence," IEEE Transactions on Control of Network Systems, vol. 1, no. 1, pp. 15-27, 2014.

[22] C. Malherbe, N. Vayatis, "Global optimization of Lipschitz functions," In Proceedings of the 34th International Conference on Machine Learning, vol. 70, pp. 2314-2323, 2017.

[23] P. Deuflhard, "Recent progress in extrapolation methods for ordinary differential equations," SIAM review, vol. 27, no. 4, pp. 505-535, 1985.

[24] P.L. Fima, "Richardson Extrapolation: The Power of the 2-gon," Mathematics TODAY, 2003. P. 159. 
[25] R.H. Byrd, "A limited memory algorithm for bound constrained optimization," SIAM Journal on Scientific Computing, vol.16, no.5, pp. 1190-1208, 1995.

[26] P. Armand, P. Ségalat. "A limited memory algorithm for inequality constrained minimization," Technical Report 2003-08, University of Limoges (France) 2003.

[27] G. Yuan, X. Lu, "An active set limited memory BFGS algorithm for bound constrained optimization," Applied Mathematical Modelling, vol. 35, no. 7, pp. 3561-3573, 2011.

[28] D.C. Liu, J. Nocedal, "On the limited memory BFGS method for large scale optimization," Mathematical Programming, vol.45, no.1-3, pp.503-528, 1989.

[29] J.C. Gilbert, C. Lemaréchal, "Some numerical experiments with variable-storage quasi-Newton algorithms," Mathematical Programming, vol.45, no.1-3, pp.407-435, 1989.

[30] Y. Li, Z. Wen, Y. Cao, Y. Tan, D. Sidorov, D. Panasetsky, "A combined forecasting approach with model self-adjustment for renewable generations and energy loads in smart community," Energy, vol. 129, pp.216-227, 2017.

[31] V.G. Kurbatsky, D.N.Sidorov, V.A. Spiryaev, N.V. Tomin, "The hybrid model based on Hilbert-Huang transform and neural networks for forecasting of short-term operation conditions of the power system," IEEE Trondheim PowerTech, Trondheim, 2011, pp. 1-7, 2011.

[32] Y. Yuan, J. Zhou, P. Ju, and J. Feuchtwang. "Probabilistic load flow computation of a power system containing wind farms using the method of combined cumulants and Gram-Charlier expansion," IET renewable power generation, vol. 5, no. 6, pp. 448-454, 2011.

[33] N. Nikmehr, S.N. Ravadanegh, "Heuristic probabilistic power flow algorithm for microgrids operation and planning," IET Generation, Transmission \&Distribution, vol. 9, no.11, pp. 985-995, 2015.

[34] G.Verbic, C.A. Canizares, "Probabilistic optimal power flow in electricity markets based on a twopoint estimate method," IEEE transactions on Power Systems, vol.21, no.4, pp. 1883-1893, 2006.

[35] "Software package for power system modeling," [Online]. Available in RUSSIAN: http://anares.ru/ software

[36] Huang C. M. et al., "Comparative study of evolutionary computation methods for active-reactive power dispatch," IET generation, transmission \& distribution, vol. 6, no. 7, pp. 636-645, 2012.

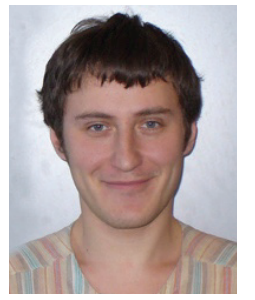

Alexander Domyshev received the M.Sc. degree in 1998 from Irkutsk State Technical University (ISTU), Irkutsk, Russia. Since 1999, he has been a researcher at MESI. He is the team leader of the ANARES package developers (anares.ru). His research interests include the optimal power flow, power system stability, emergency control, application of machine learning models to power systems, application of artificial neural network models to power systems. He is an IEEE Member.

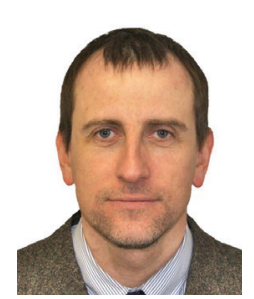

Denis N. Sidorov serves as the Leading Researcher of the Operations Research Laboratory of Energy Systems Institute of Russian Academy of Sciences. He received his degrees of Ph.D. in 1999 and Habilitation (DSc) in 2014. He is a Distinguished Guest Professor of Hunan University, Changsha, China. He has held Vision Engineer Lead position at ASTI Holdings Pte Ltd, Singapore (2005-2008), Research Fellow at Trinity College Dublin, Ireland (20012002) and CNRS, Compiegne, France (2003-2004). He is a Senior Member of IEEE, Expert of the Russian Science Foundation and the Russian Foundation for Basic Research. A reviewer of Mathematical Reviews and Zentralblatt fur Mathematik. Dr. Sidorov is an IEEE Chapter Chair of IEEE PES Russia. He serves as a Member of the Editorial Boards of Renewable and Sustainable Energy Reviews. His main research interests include machine learning, power engineering, image processing, numerical methods, and integral equations. He is a Professor of the Russian Academy of Sciences.

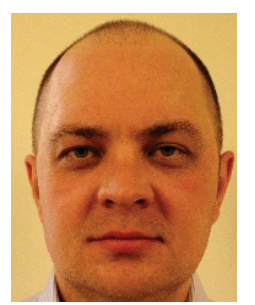

Daniil Panasetsky received the B.Sc. and Ph.D. degrees in 2006 and 2014, respectively, from Irkutsk State Technical University (ISTU) and Melentiev Energy Systems Institute (MESI), Irkutsk, Russia. Since 2009, he has been a senior researcher in MESI. Since 2014, he has been an associated professor of electrical engineering in ISTU. His research interests include power system stability, emergency control, ac/dc converters, and application of artificial intelligence to power systems. 